\title{
TP53 Arg72Pro polymorphism is associated with increased overall survival but not response to therapy in Portuguese/Caucasian patients with advanced cervical cancer
}

\author{
ANA COELHO $^{1 *}$, AUGUSTO NOGUEIRA $^{1,2^{*}}$, SÍlVIA SOARES ${ }^{1}$, JOANA ASSIS $^{1,2}$,

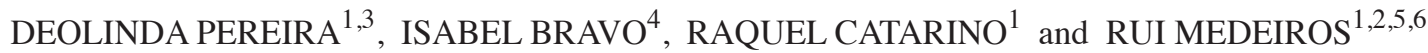

\begin{abstract}
${ }^{1}$ Molecular Oncology and Viral Pathology Group, Portuguese Oncology Institute of Porto-Research Center (CI-IPOP), Portuguese Oncology Institute of Porto (IPO-Porto), 4200-072 Porto; ${ }^{2}$ FMUP, Faculty of Medicine, University of Porto, 4200-319 Porto; ${ }^{3}$ Oncology Department, Portuguese Oncology Institute of Porto, 4200-072 Porto; ${ }^{4}$ Medical Physics,

Radiobiology and Radioprotection Group, Portuguese Oncology Institute of Porto-Research Center (CI-IPOP), Portuguese Oncology Institute of Porto, 4200-072 Porto; ${ }^{5}$ CEBIMED, Faculty of Health Sciences of Fernando Pessoa University, 4249-004 Porto; ${ }^{6}$ Research Department, Portuguese League Against Cancer (NRNorte), 4200-172 Porto, Portugal
\end{abstract}

Received September 26, 2017; Accepted February 9, 2018

DOI: $10.3892 / 01.2018 .8354$

\begin{abstract}
Identification of mechanisms that influence the therapeutic response and survival in patients with cancer is important. It is known that the genetic variability of the host, including presence of genetic polymorphisms in genes involved in DNA damage response, serves a crucial role in the prognosis of these patients. The present hospital-based retrospective cohort study aimed to evaluate the influence of TP53 Arg72Pro (rs1042522) polymorphism in the clinical outcome of 260 Caucasian patients diagnosed with cervical cancer and treated with concomitant radiotherapy and chemotherapy. The polymorphism genotyping was assessed using allelic discrimination by quantiative polymerase chain reaction. The results indicate that the TP53 Arg72Pro polymorphism did not significantly impact the response to therapy $(\mathrm{P}=0.571)$ nor disease-free survival $(\mathrm{P}=0.081)$. However, the polymorphism did influence overall survival, as increased median survival time was observed for patients carrying Arg/Pro genotype when compared with patients with Arg/Arg and Pro/Pro genotypes (126 months vs. 111 months, respectively; $\mathrm{P}=0.047$ ). To conclude, the present findings suggest that a pharmacogenomic profile based on the genetic background of patients, including the analysis of
\end{abstract}

Correspondence to: Professor Rui Medeiros, Molecular Oncology and Viral Pathology Group, Portuguese Oncology Institute of Porto-Research Center (CI-IPOP), Portuguese Oncology Institute of Porto (IPO-Porto), 62 António Bernardino de Almeida Street, 4200-072 Porto, Portugal

E-mail: ruimedei@ipoporto.min-saude.pt

*Contributed equally

Key words: cervical cancer, genetic polymorphism, TP53 Arg72Pro, treatment response, overall survival, disease-free survival the TP53 genotypes, may individualize treatment nad assist in the selection of therapies that may improve clinical outcome and lower toxicity for the patients.

\section{Introduction}

Cervical cancer is the fourth most common cancer in women, and the seventh overall, with an estimated 528,000 new cases and 266,000 deaths worldwide in 2012 (1). Currently, for locally advanced cervical cancer standard therapy is cisplatin-based concurrent chemoradiotherapy, with an overall survival (OS) of approximately $66 \%$ at 5 years (2-5).

Chemotherapy and radiotherapy are both considered as DNA damage agents, more precisely capable to introduce DNA double-strand breaks (DSBs) in order to induce cell death (6-8). When cellular DNA damage is not repaired one alternative response is apoptosis, which is the objective of the current therapeutic approach with cytotoxic agents and radiotherapy, but genetic alterations at key proteins in the pathway may result in the development of resistance to therapy $(9,10)$. Therefore, studies involving variations in genes involved in cellular response to damage are important to understand how the development of resistant phenotypes occurs. One example may be the TP53 gene, the 'guardian of genome' due to its role on cell cycle arrest, DNA repair activation and regulation of apoptosis (11-13). This suppressor gene is located on chromosome 17 (17p13.1) and encodes a phosphoprotein of 393 long amino acids $(14,15)$.

Polymorphic variants are the substitution of a single base which results in alteration of the codon may have different conformation and function, i.e., no changes cannot occur or can be gain or loss of protein function $(16,17)$. Several TP53 mutant proteins associated with tumors, have gained oncogenic function besides losing the suppressive function $(18,19)$. The most studied polymorphism of the TP53 gene is the TP53 Arg72Pro (rs1042522), which influences the protein expression of TP53 protein expression (20). This variant results from a change of guanine $(\mathrm{G})$ to cytosine $(\mathrm{C})$ in codon 72 in exon 4 , that leads 
to the replacement of arginine (Arg) by proline (Pro) (21-23). It should be noted that due to the location in the proline-rich region of the TP53 gene, this single nucleotide polymorphism (SNP) may interfere with protein stability $(24,25)$. The two allelic variants confer different susceptibilities to cancer progression, because they are structurally and functionally different (26). In studies in vivo and in vitro, the Arg allele has a higher capacity to induce apoptosis than the Pro allele. The functions associated with Pro allele include higher induction of cell cycle arrest in G1 and better activation of TP53 dependent DNA repair (27). It has also been mentioned that this polymorphism can influence the individual response (28).

Concerning cervical cancer, few studies have evaluated the predictive role of TP53 Arg72Pro polymorphism in clinical outcome and the results are contradictory $(29,30)$. Therefore, we have conducted this study to assess the possible influence of the TP53 Arg72Pro polymorphism (rs1042522) in OS and disease-free survival (DFS) in patients with advanced cervical cancer.

\section{Materials and methods}

Patients. We conducted a retrospective hospital-based study analyzing a total of 260 Caucasians patients with histologically confirmed locally advanced cervical carcinoma (FIGO stage IB2-IVA). These patients were recruited between February 2002 and October 2009, from the north region of Portugal and treated with cisplatin-based chemotherapy (40 mg/m² per week) and concomitant external radiotherapy and/or brachytherapy in Portuguese Institute of Oncology Francisco Gentil (Porto, Portugal). All women were selected consecutively according to the following inclusion criteria: Women with histological and cytology diagnosis of cervical cancer, age greater than or equal to 18 years, stage IB2-IVA and QTRT concomitant. Regarding exclusion criteria, these were surgery before treatment; absence of informed consent; failure to comply with any of the inclusion criteria.

Patients' clinical characteristics obtained from medical records are described in Table I. The median age at diagnosis was 48.00 years, the more frequent histological type was squamous cancer cell, the stage more common was IIB and the median follow up time was 63.5 months. The tumor stage was evaluated according to the International Federation of Gynecology and Obstetrics (FIGO) classification system, and the assessment of histology type was based the on system of Bethesda classification. Genomic DNA was extracted from peripheral blood samples by using FavorPrep ${ }^{\mathrm{TM}}$ Genomic DNA Mini kit (FABGK ${ }^{\circledR}$ 300; Favorgen Biotech Corp., Ping-Tung, Taiwan), according to the manufacturers protocol. All samples were obtained with the informed consent of the participants prior to their inclusion in the study, according to Helsinki Declaration principles and after approval of the Portuguese Institute of Oncology ethics committee (CES.287/014).

Evaluation of chemoradiotherapy response. The therapy response was evaluated according to RECIST criteria (31). Complete response (CR) indicates disappearance of the disease, partial response (PR) indicates at least $50 \%$ reduction in tumor load, stable disease (SD) indicates that the lesion showed $\leq 25 \%$ progression or $<50 \%$ shrinkage, and progression of disease (PD) indicates $>25 \%$ enlargement of the lesion, or appearance of a new lesion. $\mathrm{CR}$ and $\mathrm{PR}$ were considered to be a good response; $\mathrm{SD}$ and $\mathrm{PD}$, a poor response.

Genotyping of TP53 Arg72Pro (rs1042522) polymorphism. The selected SNP was chosen from the best evidence from published studies $(29,30,32,33)$, through public databases who provide information on the phenotypic risks, had a minor allele frequency of an at least 10 to $20 \%$ and the SNP biological effect. The genotyping was performed using Taqman ${ }^{\mathrm{TM}}$ Allelic Discrimination methodology by quantitative polymerase chain reaction (qPCR). This method uses probes labeled with fluorochromes specific for each allele, thus VIC probe is allele $\mathrm{C}$ and the FAM probe is allele $\mathrm{G}$ (AGGAGCTGCTGGvTGC AGGGGCCACG [C/G] GGGGAGCAGCCTCTGGCATTC TGGG). The allelic discrimination PCR reactions were carried out in $6 \mu \mathrm{l}$ volumes using $2.5 \mu \mathrm{l}$ of TaqMan ${ }^{\circledR}$ Universal PCR Master Mix (2X), $0.125 \mu \mathrm{l}$ of 40x assay mix $2.375 \mu \mathrm{l}$ of sterile $\mathrm{H}_{2} \mathrm{O}$ and $1 \mu \mathrm{l}$ of genomic DNA. Amplification of DNA was carried out using the following amplification conditions: $95^{\circ} \mathrm{C}$ for $10 \mathrm{~min}$, followed by 45 cycles of $95^{\circ} \mathrm{C}$ for $15 \mathrm{sec}$ and $60^{\circ} \mathrm{C}$ for $1 \mathrm{~min}$. Data capture and analysis was carried thought the ABI 7300 Real Time PCR System (Applied Biosystems; Thermo Fisher Scientific, Inc., Waltham, MA, USA) and the Sequence Detection Systems software (version 1.2.3; Applied Biosystems; Thermo Fisher Scientific, Inc.).

Quality control included the use of negative controls in all runs, double sampling in at least $10 \%$ of the samples, genotyping performed blindly regarding to clinical and pathologic characteristics of patients and the results independently evaluated by two researchers. We observed complete concordance among duplicates.

Statistical analysis. Difference in frequencies of the TP53 Arg72Pro genotypes between the different chemoradiotherapy responses groups were evaluated by $\chi^{2}$ test. The OS and the overall survival at 5 years was defined from the date of diagnosis to the date of death and the percentage of patients alive after 5 years of diagnosis, respectively. The DFS times were defined from the data from the date of diagnosis to the date of disease recurrence. Patients without progression, lost to follow-up or died from other causes were censored at their last date of record. In the evaluation of OS and DFS was used Kaplan-Meier survival estimate and log-rank test. We applied a multivariate analysis using $\mathrm{COX}$ regression method to calculate hazard ratio (HR) and 95\% confidence intervals (CI) for the association between the genotypes and the risk of death in advanced cervical cancer patients. This analysis was used to adjust for potential confounders, such as age ( $<48$ years vs. $\geq 48$ years), stage ( $<$ IIB vs. $\geq$ IIB), smoking habits (non-smokers vs. smokers and former smokers) and histological type (adenosquamous cell carcinomas and small cell carcinoma vs. adenocarcinoma and squamous cell carcinoma), with TP53 Arg72Pro genotypes fitted as indicator variables. A level of $\mathrm{P}<0.05$ was considered statistically significant. All analysis of data was performed using the computer software Statistical Package for Social Sciences (SPSS) for Windows (version 22.0; IBM Corp., Armonk, NY, USA).

As our study was performed based on DNA availability, we did not carry out any power analysis before the study. 
Table I. Distribution of patients' clinicopathologic characteristics.

Characteristics

Age (years)

Median, 48.00

Mean \pm SD, $49.00 \pm 11.50$

Follow-up time (months)

Median, 63.5 (range 3-115)

Number of chemotherapy cycles

Median, 6 (range 1-6)

Total dose of radiotherapy (Gy)

Median, 80 (range 45-88)

Tumor stage

IB2

IIA2

IIB

IIIA

IIIB

IVA

Histologic type

Squamous cell carcinoma

$216(83.1)$

Adenocarcinoma

5 (1.9)

Small cell carcinoma

Not present

Unknown

Progression

$210(80.8)$

SD, standard deviation.

Therefore, we cannot report on any original study power. However, two-way analysis of variance followed by post hoc analysis was performed as follows: The power to detect a hazard ratio of 2.001 obtained by multivariate analysis, depended on the distribution of the polymorphism genotypes, patients' median survival time, recruitment period (93 months) and additional follow-up time (63.5 months). Consequently, assuming a type I error probability of 0.05 , we estimate a post hoc power higher than $80 \%$. This analysis was performed using the Power and Sample Size program (version 3.1.2).

\section{Results}

As mentioned before, the TP53 Arg72Pro polymorphism studied in this work results from a change of guanine $(\mathrm{G})$ to cytosine $(\mathrm{C})$ in codon 72 in exon 4 that leads to the replacement of arginine (Arg) by proline (Pro). Besides that, all results are presented for an analysis comparing heterozygote genotypes (Arg/Pro) with homozygous genotypes (Arg/Arg and Pro/Pro).

Of the 260 patients included in this study (Table I), only 249 patients have results for genotyping. The frequencies of Arg/Arg, Arg/Pro and Pro/Pro genotypes were 0.10, 0.33 and 0.56, respectively. The allele frequency for Arg allele and Pro allele was 27.11 and $72.89 \%$, respectively. The good treatment response rate was 10.7, 33.9 and 55.4\% for Arg/Arg, Arg/Pro and Pro/Pro genotypes, respectively. Poor treatment response rate for Arg/Arg, Arg/Pro and Pro/Pro genotypes was 6.3, 25.0 and $68.8 \%$, respectively. This polymorphism were found to be not associated with response to therapy $(\mathrm{P}=0.571)$ (Table II).

Regarding OS rates found using Kaplan-Meier method and log-rank test, we observed that the mean survival rates were not statistically different according to the patients TP53 Arg72Pro genotypes $(\mathrm{P}=0.058)$, age $(\mathrm{P}=0.630)$ and histology $(\mathrm{P}=0.758)$. Stage $(\mathrm{P}=0.008)$ and recurrence $(\mathrm{P}<0.001)$ were independent prognostic factors that influenced significantly OS of women with advanced cervical cancer treated with chemoradiotherapy (QTRT). Moreover, there are significant differences in mean survival between heterozygote genotypes (Arg/Pro) and homozygous genotypes (Arg/Arg and Pro/Pro). The group of patients carrying heterozygous genotype present a higher mean survival rate than the other patients (126 vs. 111 months, $\mathrm{P}=0.047$ ) (Fig. 1).

Concerning smoking history, our results demonstrate that OS time differed according to the Arg/Arg and Pro/Pro homozygous genotype vs Arg/Pro heterozygous genotypes carriers in non-smoker individuals, but these results are in the threshold for statistical significance ( $\mathrm{P}=0.052$; Fig. $2 \mathrm{~A})$. No statistically significant differences were found in the genotype frequencies and OS rate among smokers and former smokers $(\mathrm{P}=0.194$; Fig. 2B). Using the Cox regression analysis, we found that carriers of TP53 Arg72Pro homozygous genotypes (Arg/Arg and Pro/Pro) present a 2-fold increase of risk of death which is not statistically significant, when compared with TP53 Arg72Pro heterozygous genotypes, with tumor stage, median age, histology and smoking history as covariates [hazard ratio (HR), 2.001; 95\% CI, 0.917-4.368; $\mathrm{P}=0.082$ ] (Table III).

No difference was found for DFS according to the distribution of genotypes of TP53 Arg72Pro polymorphism ( $\mathrm{P}=0.205)$, same when comparing patients with heterozygous and homozygous genotypes $(\mathrm{P}=0.081$; Fig. 3$)$.

\section{Discussion}

The activation of the response to DNA damage aims to cell cycle arrest and DNA repair, and the lesions correction failure can result in the senescence or apoptosis (34). Assuming that cells respond differently to DNA damage taking into account whether or not they are tumor cells, understanding this mechanisms will allow selection of therapeutic strategies to individualize response to DNA damage in altered in 
Table II. Response to treatment of the advanced cervical cancer patients treated with chemoradiotherapy according to genotypes of the TP53 Arg72Pro polymorphism.

\begin{tabular}{lcc}
\hline TP53 Arg72Pro polymorphism & Good response $(\mathrm{CR}+\mathrm{PR})$, no $(\%)$ & Poor response (SD + PD), no. (\%) \\
\hline Genotype & & $1(6.3)$ \\
Arg/Arg & $25(10.7)$ & $4(25.0)$ \\
Arg/Pro & $79(33.9)$ & $11(68.8)$ \\
Pro/Pro & $129(55.4)$ & $6(18.8)$ \\
Allele & & $26(81.2)$ \\
Arg & $129(27.7)$ & 0.271 \\
Pro & $337(72.3)$ & 260 \\
\hline
\end{tabular}

$\mathrm{CR}$, complete response; $\mathrm{PR}$, partial response; $\mathrm{SD}$, stable disease; $\mathrm{PD}$, progression disease.

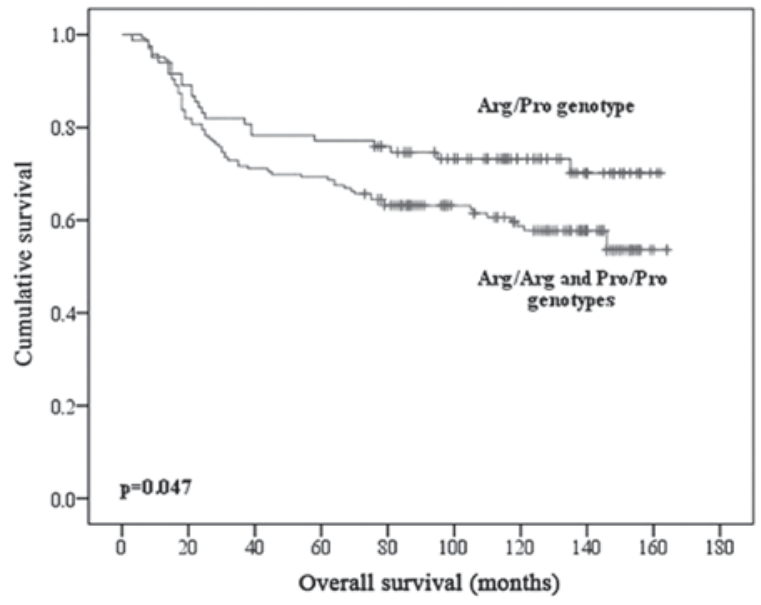

Figure 1. Overall survival by Kaplan-Meier method and log-rank test of cervical cancer patients according to TP53 Arg72Pro polymorphism.

cancer cells (35). Apoptosis is the main mechanism by which anti-cancer agents originate toxicity (36).

One major mechanism of resistance to therapy and cell survival is the inactivation of the function of TP53 gene, so observed resistance in tumor cells harboring wild type TP53 for a large variety of agents, such as ionizing radiation and several classes of cytotoxic drugs, can occur directly by factors that regulate or negate the functional activity of this protein, or indirectly, by deregulation of pathways downstream of this gene (37).

The role of the TP53 Arg72Pro polymorphism remains controversial (17). The segregation of this polymorphism shows pronounced ethnic differences, so results will be dependent of the study population $(32,38)$. It is also important to refer that in this study we did not consider HPV infections, although it might be a relevant issue for additional studies. However, Medeiros and colleagues (39) studied HPV genotyping profile in squamous cervical lesions in Portugal, and then they find high prevalence of HPV-16 and -18, approximately 80 and $15 \%$, respectively, in cases with invasive cervical cancer. Thus, future studies may include HPV genotyping to evaluate its role in disease progression and clinical outcome under the influence of the genetic background.

In ourstudy, carriers of the heterozygous genotype (Arg/Pro) had a higher mean survival overall than patients with both homozygous genotypes (Arg/Arg and Pro/Pro). Several studies have evaluated the influence of TP53 codon 72 polymorphism in the clinical outcome of cancer patients with controversial results $(29,30,33,40)$. Investigation of Piña-Sánchez et al (30) in Mexican women with cervical cancer found a higher survival in heterozygous women (Arg/Pro) than in homozygous women (Arg/Arg and Pro/Pro), however without significant statistical differences. In study of Liu et al (29), they did not find association of TP53 Arg72Pro polymorphism and clinical outcome in Chinese women with cervical carcinoma.

In patients with pancreatic, testicular and prostate cancer no significant effect of this polymorphism was found $(40,41)$. Pro allele homozygosis has been linked with lower sensitivity to chemotherapy in breast and head and neck cancer and lower survival in breast, lung and colorectal cancer (42-46). Moreover other studies show that carriers of Arg genotype have a higher treatment response rate and survival after chemoradiotherapy in advanced head and neck cancer and lung and breast cancer $(43,44,47)$. The presence of one mutated Arg allele may be associated with a reduced sensitivity to cancer therapy in head and neck cancer as well as retention of the Arg allele in heterozygous women with breast cancer are associated with a reduced OS and progression-free disease (48). In this sense, Sullivan and colleagues (44) found that drugs exert their cytotoxic effect in different ways, according to the two codon 72 mutant variant of TP53 gene, thus verifying a differentiated cell resistance.

There are four possible reasons that may explain the fact that homozygous patients had lower survival than heterozygous patients: i) As seen in other types of cancer, patients with homozygous Pro allele have lower survival than heterozygous carriers, since this allele has a major role in cell cycle arrest and DNA repair than Arg allele (27). Wild type TP53 Pro variant activates several genes involved in DNA repair more effectively than TP53 Arg variant. At the same time, cells expressing the TP53 Pro allele were able to repair the DNA damage much more effectively than cells expressing TP53 Arg allele (25); ii) Patients with homozygous Arg allele showed lower survival rates compared to heterozygous. It is important refer that the Arg variant has been correlated with a higher affinity of binding and degradation of TP53 protein by E6 oncoprotein of HPV-16/HPV-18 $(38,49-51)$. One of the better well-known functions of the HPV E6 is the ability to 
Table III. Multivariate analysis of death risk at 5 years by Cox regression for the TP53 genotypes, adjusted to different clinical and pathological variables.

\begin{tabular}{lll}
\hline Clinicopathological characteristics & HR & 95\% CI \\
\hline Median age (<48 years/ $\geq 48$ years) & 1.319 & $0.693-2.511$ \\
Tumor stage (<IIB/ $\geq \mathrm{IIB})$ & 3.212 & $0.767-13.445$ \\
Tobacco (non-smokers/smokers and former smokers) & 0.938 & 0.400 \\
Histology (adenosquamous cell carcinomas and & 0.680 & $0.092-5.001$ \\
small cell carcinoma/adenocarcinoma and & & 0.364 \\
squamous cell carcinoma) & 2.001 & 0.705 \\
TP53 Arg72Pro genotypes & & $0.917-4.368$ \\
(Homozygous/heterozygous) & & 0.082 \\
\hline
\end{tabular}

HR, hazard ratio; CI, confidence interval.
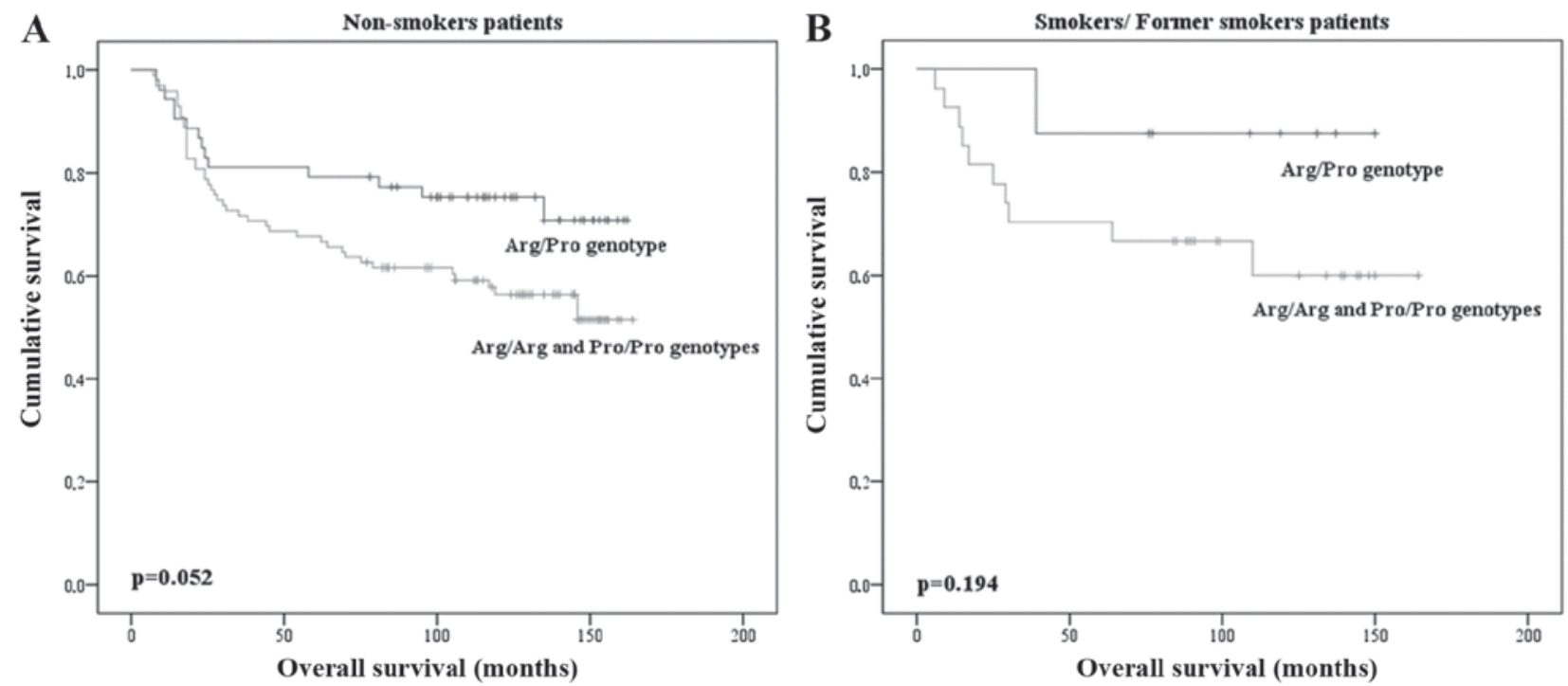

Figure 2. Overall survival by Kaplan-Meier method and log-rank test of cervical cancer patients according to TP53 Arg72Pro genotypes, adjusted to smoking habits: (A) Non-smokers group; (B) smokers/former smokers group.

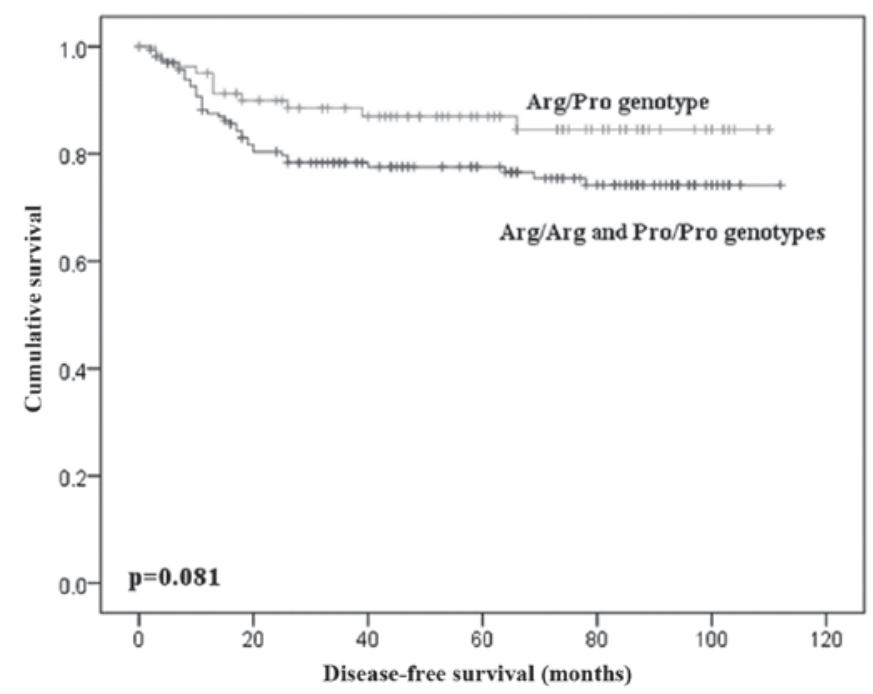

Figure 3. Disease-free survival by Kaplan-Meier method and log-rank test in cervical cancer patients according to genotypes of TP53 Arg72Pro polymorphism. increase the tolerance of normal response to DNA damage or then the independent regulation of cellular growth $(52,53)$. These actions are promoted by TP53 degradation as well as the inhibition of the gene and the multiple repair pathways. Furthermore, the expression of E6 decreases the ability to repair DSBs (53). In this sense, it is believed that carriers of the Arg/Arg homozygous genotype have lower apoptotic capacity, which results in poor survival; iii) the low survival for Arg/Arg homozygous patients have a greater affinity for the mutant TP53 creating mutants with gain-of-function. Furthermore this variant seems to inhibit the pro-apoptotic activity of the TP73 gene, which determines the cellular response to different anticancer drugs. In head and neck tumors where TP53 gene is frequently mutated it is noted that Arg allele carriers had higher resistance to chemotherapy leads to shorter survival. It should be noted that the Pro allele is more frequent with TP53 wild type and tumors are less sensitive to apoptosis (25); d) it is known that tumors with Arg allele were associated with insufficient or absence of apoptosis, because it was observed the absence of coexpression of Fas and FasL, as well as 
high expression of Bcl-2 protein. In heterozygous carriers of Arg72Pro polymorphism the absence of expression of Bcl-2 and co-expression of Fas/FasL is not found. The Bcl-2, Fas and FasL are three apoptosis-related proteins and the down regulation of Fas expression is common in vary type of cancers, including gynecological cancers (54).

This study also indicates that the influence of Arg72Pro polymorphism in treatment response of cervical cancer patients seems to be modulated by smoking history. Our results demonstrate that non-smoker carriers of homozygous genotype present a lower mean OS time comparing with patients with heterozygous genotype $(\mathrm{P}=0.052)$, but these results are in the threshold for statistical significance. However, this potential association was not observed in smoker or former-smokers $(\mathrm{P}=0.194)$. In a similar study in lung cancer no association was found between genotype of the polymorphism and OS of patients, taking into account their smoking history $(\mathrm{P}=0.850)(55)$. Moreover, the biological mechanism that may explain these differences in results is not yet known.

One of the possible limitations of the present study was the exclusion of participants with cervical intraepithelial neoplasia (CIN) or uninfected controls. Therefore, future studies including these types of participants will be relevant to make this study more complete, so to compare the impact of the TP53 Arg72Pro polymorphism in clinical outcome between pre-invasive cancer patients and advanced cervical cancer patients.

In conclusion, our results demonstrate survival advantage in heterozygous carriers of Arg72Pro polymorphism and a trend to greater risk of death in the homozygous carriers of this polymorphism. Furthermore, TP53 genotypes could be a useful molecular tools for predicting the clinical outcome of cervical cancer patients and may allow to evaluate optional therapeutic regimens in patients with lower survival. Therefore, in the attempt of optimizing responses and minimizing toxicities associated with chemoradiotherapy, the analysis of a wide range of genetic polymorphisms in DNA damages response genes may indicate the more suitable therapeutic procedure for each cancer patient.

\section{Acknowledgements}

This authors thank the Research Department-Portuguese League against Cancer (NRNorte) for their general support provided.

\section{Funding}

The present study was funded by a research grant for Doctoral project of Augusto Nogueira from the Ministério da Ciência, Tecnologia e Ensino Superior-FCT (Fundação para a Ciência e a Tecnologia: SFRH/BD/124155/2016) and by Project no. CI-IPOP-22-2015 from the Research Center the Portuguese Institute of Oncology of Porto, (Porto, Portugal).

\section{Availability of data and materials}

The datasets used and/or analysed during the current study are available from the corresponding author on reasonable request.

\section{Authors' contributions}

$\mathrm{AC}$ and $\mathrm{AN}$ conceived and designed the experiments. $\mathrm{AC}$ and AN performed the experiments. AC, AN and RM analyzed the data. SS, JA, IB and RC contributed to the interpretation of results obtained and manuscript construction. DP analyzed and interpreted the patients' data regarding the clinical characteristics. AC and AN wrote the paper. All authors read and approved the final manuscript.

\section{Ethics approval and consent to participate}

All samples were obtained with the informed consent of the participants prior to their inclusion in the study, according to Helsinki Declaration principles and after approval of the ethics committee of Portuguese Oncology Institute of Porto (CES.287/014).

\section{Consent for publication}

Not applicable.

\section{Competing interests}

The authors declare that they have no competing interests.

\section{References}

1. Ferlay J, Soerjomataram I, Dikshit R, Eser S, Mathers C, Rebelo M, Parkin DM, Forman D and Bray F: Cancer incidence and mortality worldwide: Sources, methods and major patterns in GLOBOCAN 2012. Int J Cancer 136: E359-E386, 2015.

2. Noordhuis MG, Eijsink JJH, Roossink F, de Graeff P, Pras E, Schuuring E, Wisman GB, de Bock GH and van der Zee AG: Prognostic cell biological markers in cervical cancer patients primarily treated with (chemo)radiation: A systematic review. Int J Radiat Oncol Biol Phys 79: 325-334, 2011.

3. Downs L: Advances in cervical cancer treatment. Gynecol Oncol 121: 431-433, 2011.

4. Fujiwara M, Isohashi F, Mabuchi S, Yoshioka Y, Seo Y, Suzuki O, Sumida I, Hayashi K, Kimura T and Ogawa K: Efficacy and safety of nedaplatin-based concurrent chemoradiotherapy for FIGO Stage IB2-IVA cervical cancer and its clinical prognostic factors. J Radiat Res 56: 305-314, 2015.

5. Tangjitgamol S, Katanyoo K, Laopaiboon M, Lumbiganon P, Manusirivithaya S and Supawattanabodee B: Adjuvant chemotherapy after concurrent chemoradiation for locally advanced cervical cancer. Cochrane Database Syst Rev 3: CD010401, 2014.

6. Hosoya $\mathrm{N}$ and Miyagawa K: Targeting DNA damage response in cancer therapy. Cancer Sci 105: 370-388, 2014.

7. Bajinskis A, Natarajan AT, Erixon K and Harms-Ringdahl M: DNA double strand breaks induced by the indirect effect of radiation are more efficiently repaired by non-homologous end joining compared to homologous recombination repair. Mutat Res 756: 21-29, 2013.

8. Goldstein $\mathrm{M}$ and Kastan MB: The DNA damage response: Implications for tumor responses to radiation and chemotherapy. Annu Rev Med 66: 129-143, 2015.

9. Khan KH, Blanco-Codesido M and Molife LR: Cancer therapeutics: Targeting the apoptotic pathway. Crit Rev Oncol Hematol 90: 200-219, 2014.

10. Jekimovs C, Bolderson E, Suraweera A, Adams M, O'Byrne KJ and Richard DJ: Chemotherapeutic compounds targeting the DNA double-strand break repair pathways: The good, the bad and the promising. Front Oncol 4: 86, 2014.

11. Basu A and Krishnamurthy S: Cellularresponses to Cisplatin-induced DNA damage. J nucleic acids 2010: 201367, 2010.

12. Zhou X, Gu Y and Zhang SL: Association between p53 codon 72 polymorphism and cervical cancer risk among Asians: A huge review and meta-analysis. Asian Pac J Cancer Prev 13: 4909-4914, 2012. 
13. Sionov RV and Haupt Y: The cellular response to p53: The decision between life and death. Oncogene 18: 6145-6157, 1999.

14. Naccarati A, Polakova V, Pardini B, Vodickova L, Hemminki K, Kumar R and Vodicka P: Mutations and polymorphisms in TP53 gene-an overview on the role in colorectal cancer. Mutagenesis 27: 211-218, 2012.

15. Golubovskaya VM and Cance WG: Targeting the p53 pathway Surg Oncol Clin N Am 22: 747-764, 2013.

16. Dos Santos HG, Nunez-Castilla J and Siltberg-Liberles $\mathbf{J}$ : Functional diversification after gene duplication: Paralog specific regions of structural disorder and phosphorylation in p53, p63 and p73. PLoS One 11: e0151961, 2016.

17. Donehower LA: p53: Guardian and suppressor of longevity? Exp Gerontol 40: 7-9, 2005

18. Haupt S, Raghu D and Haupt Y: Mutant p53 drives cancer by subverting multiple tumor suppression pathways. Front Oncol 6 : $12,2016$.

19. Liu J, Zhang C and Feng Z: Tumor suppressor p53 and its gain-of-function mutants in cancer. Acta biochim biophys Sin (Shanghai) 46: 170-179, 2014

20. Dastjerdi MN: TP53 codon 72 polymorphism and P53 protein expression in colorectal cancer specimens in Isfahan. Acta Med Iran 49: 71-77, 2011

21. Hu X, Zhang Z, Ma D, Huettner PC, Massad LS, Nguyen L, Borecki I and Rader JS: TP53, MDM2, NQO1 and susceptibility to cervical cancer. Cancer Epidemiol Biomarkers Prev 19: 755-761, 2010.

22. Santos AM, Sousa H, Catarino R, Pinto D, Pereira D, Vasconcelos A, Matos A, Lopes C and Medeiros R: TP53 codon 72 polymorphism and risk for cervical cancer in Portugal. Cancer Genet Cytogenet 159: 143-147, 2005

23. Dokianakis DN and Spandidos DA: P53 codon 72 polymorphism as a risk factor in the development of HPV-associated cervical cancer. Mol Cell Biol Res Commun 3: 111-114, 2000.

24. Sousa H, Santos AM, Pinto D and Medeiros R: Is there a biological plausability for p53 codon 72 polymorphism influence on cervical cancer development? Acta Med Port 24: 127-134, 2011.

25. Hrstka R, Coates PJ and Vojtesek B: Polymorphisms in p53 and the p53 pathway: Roles in cancer susceptibility and response to treatment. J Cell Mol Med 13: 440-453, 2009.

26. Dahabreh IJ, Schmid CH, Lau J, Varvarigou V, Murray S and Trikalinos TA: Genotype misclassification in genetic association studies of the rs1042522 TP53 (Arg72Pro) polymorphism: A systematic review of studies of breast, lung, colorectal, ovarian and endometrial cancer. Am J Epidemiol 177: 1317-1325, 2013.

27. Bojesen SE and Nordestgaard BG: The common germline Arg72Pro polymorphism of p53 and increased longevity in humans. Cell Cycle 7: 158-163,2008.

28. Bergamaschi D, Gasco M, Hiller L, Sullivan A, Syed N, Trigiante G, Yulug I, Merlano M, Numico G, Comino A, et al: p53 polymorphism influences response in cancer chemotherapy via modulation of p73-dependent apoptosis. Cancer Cell 3: 387-402, 2003

29. Liu JH, Xi P, Chai YL, Wang J, Wang T, Liu Z and Dai PG: Association of DNA repair gene polymorphisms with response to cisplatin-based concurrent chemoradiotherapy in patients with cervical carcinoma. DNA Repair (Amst) 41: 69-72, 2016.

30. Piña-Sánchez P, Hernández-Hernández DM, Taja-Chayeb L, Cerda-Flores RM, González-Herrera AL, Rodea-Avila C, Apresa-García T, Ostrosky-Wegman P, Vázquez-Ortíz G, Mendoza-Lorenzo P, et al: Polymorphism in exon 4 of TP53 gene associated to HPV 16 and 18 in Mexican women with cervical cancer. Med Oncol 28: 1507-1513, 2011.

31. Eisenhauer EA, Therasse P, Bogaerts J, Schwartz LH, Sargent D, Ford R, Dancey J, Arbuck S, Gwyther S, Mooney M, et al: New response evaluation criteria in solid tumours: Revised RECIST guideline (version 1.1). Eur J Cancer 45: 228-247, 2009.

32. Sousa H, Santos AM, Pinto D and Medeiros R: Is the p53 codon 72 polymorphism a key biomarker for cervical cancer development? A meta-analysis review within European populations. Int J Mol Med 20: 731-741, 2007.

33. Papadakis ED, Soulitzis N and Spandidos DA: Association of p53 codon 72 polymorphism with advanced lung cancer: The Arg allele is preferentially retained in tumours arising in Arg/Pro germline heterozygotes. Br J Cancer 87: 1013-1018, 2002.

34. Hollingworth R and Grand RJ: Modulation of DNA damage and repair pathways by human tumour viruses. Viruses 7: 2542-2591, 2015.

35. Rassool FV and Tomkinson AE: Targeting abnormal DNA double strand break repair in cancer. Cell Mol Life Sci 67 3699-3710, 2010
36. Johnstone RW, Ruefli AA and Lowe SW: Apoptosis: A link between cancer genetics and chemotherapy. Cell 108: 153-164, 2002.

37. Martinez-Rivera M and Siddik ZH: Resistance and gain-of-resistance phenotypes in cancers harboring wild-type p53. Biochem Pharmacol 83: 1049-1062, 2012.

38. Petitjean A, Achatz MI, Borresen-Dale AL, Hainaut P and Olivier M: TP53 mutations in human cancers: Functional selection and impact on cancer prognosis and outcomes. Oncogene 26 : 2157-2165, 2007.

39. Medeiros R, Prazeres H, Pinto D, Macedo-Pinto I, Lacerda M, Lopes C and Cruz E: Characterization of HPV genotype profile in squamous cervical lesions in Portugal, a southern European population at high risk of cervical cancer. Eur J Cancer Prev 14 467-471, 2005

40. Dong M, Nio Y, Yamasawa K, Toga T, Yue L and Harada T: p53 alteration is not an independent prognostic indicator, but affects the efficacy of adjuvant chemotherapy in human pancreatic cancer. J Surg Oncol 82: 111-120, 2003.

41. Wu WJ, Kakehi Y, Habuchi T, Kinoshita H, Ogawa O, Terachi T, Huang $\mathrm{CH}$, Chiang $\mathrm{CP}$ and Yoshida O: Allelic frequency of p53 gene codon 72 polymorphism in urologic cancers. Jpn J Cancer Res 86: 730-736, 1995 .

42. Wang YC, Chen CY, Chen SK, Chang YY and Lin P: p53 codon 72 polymorphism in Taiwanese lung cancer patients: Association with lung cancer susceptibility and prognosis. Clin Cancer Res 5: 129-134, 1999.

43. Xu Y, Yao L, Ouyang T, Li J, Wang T, Fan Z, Lin B, Lu Y and Xie Y: p53 Codon 72 polymorphism predicts the pathologic response to neoadjuvant chemotherapy in patients with breast cancer. Clin Cancer Res 11: 7328-7333, 2005.

44. Sullivan A, Syed N, Gasco M, Bergamaschi D, Trigiante G, Attard M, Hiller L, Farrell PJ, Smith P, Lu X and Crook T: Polymorphism in wild-type p53 modulates response to chemotherapy in vitro and in vivo. Oncogene 23: 3328-3337, 2004

45. Tommiska J, Eerola H,Heinonen M, Salonen L, Kaare M, Tallila J, Ristimäki A, von Smitten K, Aittomäki K, Heikkilä P, et al: Breast cancer patients with p53 Pro72 homozygous genotype have a poorer survival. Clin Cancer Res 11: 5098-5103, 2005.

46. Starinsky S, Figer A, Ben-Asher E, Geva R, Flex D, Fidder HH, Zidan J, Lancet D and Friedman E: Genotype phenotype correlations in Israeli colorectal cancer patients. Int J Cancer 114: 58-73, 2005.

47. Pietsch EC, Humbey O and Murphy ME: Polymorphisms in the p53 pathway. Oncogene 25: 1602-1611, 2006.

48. Bonafé M, Ceccarelli C, Farabegoli F, Santini D, Taffurelli M, Barbi C, Marzi E, Trapassi C, Storci G, Olivieri F and Franceschi C: Retention of the p53 codon 72 arginine allele is associated with a reduction of disease-free and overall survival in arginine/proline heterozygous breast cancer patients. Clin Cancer Res 9: 4860-4864, 2003.

49. Storey A, Thomas M, Kalita A, Harwood C, Gardiol D Mantovani F, Breuer J, Leigh IM, Matlashewski G and Banks L: Role of a p53 polymorphism in the development of human papillomavirus-associated cancer. Nature 393: 229-234, 1998.

50. Chansaenroj J, Theamboonlers A, Junyangdikul P, Swangvaree S, Karalak A, Chinchai T and Poovorawan Y: Polymorphisms in TP53 (rs1042522), p16 (rs11515 and rs3088440) and NQO1 (rs1800566) genes in thai cervical cancer patients with HPV 16 infection. Asian Pac J Cancer Prev 14: 341-346, 2013.

51. Santos AM, Sousa H, Pinto D, Portela C, Pereira D, Catarino R, Duarte I, Lopes C and Medeiros R: Linking TP53 codon 72 and P21 nt590 genotypes to the development of cervical and ovarian cancer. Eur J Cancer 42: 958-963, 2006.

52. Peralta-Zaragoza O, Bermúdez-Morales VH, Pérez-Plasencia C, Salazar-León J, Gómez-Cerón C and Madrid-Marina V: Targeted treatments for cervical cancer: A review. Onco Targets Ther 5: $315-328,2012$

53. Wallace NA and Galloway DA: Manipulation of cellular DNA damage repair machinery facilitates propagation of human papillomaviruses. Semin Cancer Biol 26: 30-42, 2014

54. Schneider-Stock R, Mawrin C, Motsch C, Boltze C, Peters B Hartig R, Buhtz P, Giers A, Rohrbeck A, Freigang B and Roessner A: Retention of the arginine allele in codon 72 of the p53 gene correlates with poor apoptosis in head and neck cancer. Am J Pathol 164: 1233-1241, 2004

55. Matakidou A, El Galta R, Webb EL, Rudd MF, Bridle H, Eisen T and Houlston RS; GELCAPS Consortium: Lack of evidence that p53 Arg72Pro influences lung cancer prognosis: An analysis of survival in 619 female patients. Lung Cancer 57: 207-212, 2007. 